\title{
Nystagmus as the presentation of tentorial incisure subdural haematoma
}

\author{
Ely Ashkenazi, Shlomo Pomeranz
}

\begin{abstract}
A case of chronic subdural haematoma in the posterior fossa, secondary to anticoagulant treatment, is presented. The unusual clinical presentation of vertigo and nystagmus and the atypical CT are discussed. This seems to be the first report to describe a chronic subdural haematoma in the posterior fossa in an alive adult.
\end{abstract}

$(\Im$ Neurol Neurosurg Psychiatry 1994;57:830-831)

Chronic subdural haematoma is a well known clinical entity, with an incidence of one to two per 100000 people a year. ${ }^{12}$ Most patients are 50 years of age or older. Those with a history of trauma have usually had a mild or trivial head injury. A significant proportion of patients are predisposed to subdural haematoma because of chronic alcoholism, epilepsy, or coagulopathies. ${ }^{1}$ Vertigo and nystagmus are common presenting symptoms and signs in otolaryngology with a large range of aetiologies. ${ }^{3} \mathrm{We}$ present a rare case of subdural haematoma in the posterior fossa in a patient treated with anticoagulants, who presented with vertigo and nystagmus.

\section{Case report}

A 65 year old woman was admitted to the otolaryngology department because of severe true vertigo with a duration of 48 hours. She was known to have had rheumatic heart disease and underwent an aortic valve replacement 18 months before her current admission. Since then the patient had taken an oral anticoagulant, warfarin (Coumadin) (prothrombin time $40 \%-50 \%$ ), and dipyridamole (Cardoxin), an antiplatelet aggregation medication. On admission the patient was fully conscious and had severe vertigo and prominent gross horizontal nystagmus in all ocular positions. There were no other neurological deficits. Brain CT demonstrated a space occupying lesion of the left tentorial incisure $(3 \times 5 \mathrm{~cm})$ compressing the cerebellum and brain stem. Neither the peribrainstem cisterns. nor the fourth ventricle were visible due to their prominent compression. A fluid within the mass, with hyperdense and hypodense compartments, was seen (fig 1).

The patient was referred to the neurosurgical department. After correction of her coagulation profile with fresh frozen plasma and vitamin $K$, the patient underwent a suboccipital craniectomy, and a large subacute subdural haematoma was evacuated from the underside of the tentorium and the left tentorial incisure.

Postoperatively the vertigo and nystagmus resolved and the patient started to walk without assistance within 24 hours. A postoperative CT showed a normal fourth ventricle and cisterns (fig 2) with some residual air bubbles in the posterior fossa.

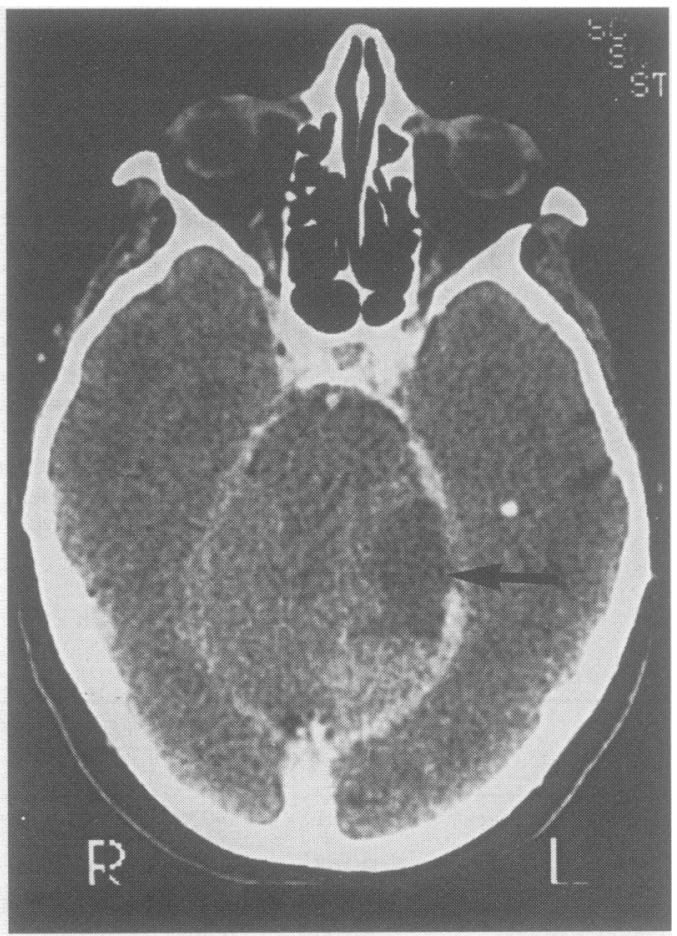

Figure 1 Axial CT at the level of the tentorial incisure demonstrating chronic subdural haematoma that has settled into a hyperdense lower component and a hypodense upper component. The fourth ventricle and cisterns surrounding the brainstem have been prominently compressed and the brainstem shifted laterally by the haematoma. 
Figure 2 Axial CT at the level of the tentorial incisure in the same patient as in fig 1 after surgical drainage of the subdural haematoma. The fourth ventricle and cisterns are easily seen. The brainstem is central. Because of $a$ different angle of the tomogram relative to fig 1 , anterior and posterior structures are different from the previous examination.

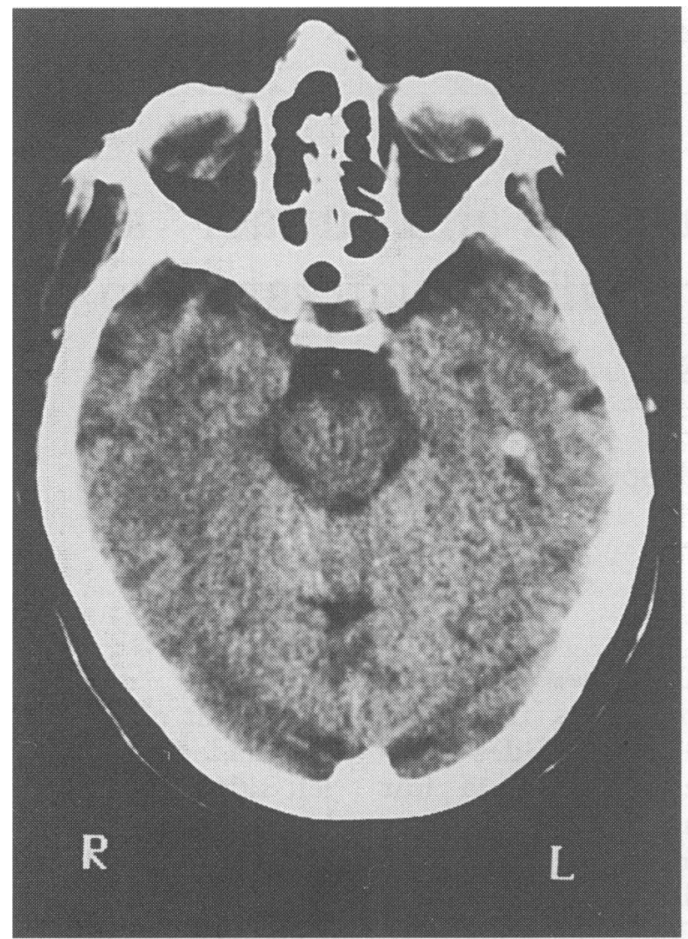

\section{Discussion}

Dizziness and vertigo are common problems seen by both otolaryngologists and neurologists. True vertigo implies that a vestibular abnormality is present. This may be due to a central abnormality emanating from the vestibular connections in the CNS (vestibular nuclei, tracts in the brainstem, cerebellum, or cerebellar outflow tracts). Patients with tumours compressing the pontine region of the brainstem may present with vertigo and nystagmus, but this is uncommon and the symptoms are usually gradually progressive. Vertical nystagmus and other cranial nerve abnormalities are often seen in central vertigo. $^{3}$

Chronic subdural haematoma is a well known entity. ${ }^{1-6}$ Posterior fossa subdural haematoma is, however, uncommon, although it has been described in infants. ${ }^{7-9}$ As far as we know no cases of infratentorial subdural haematoma in live adults have been described. Munro, in 1934, cites one case of posterior fossa subdural haematoma found at necropsy at Guy's hospital in an unoperated adult. ${ }^{410}$

Anticoagulant treatment is a predisposing factor for subdural haematoma formation, ${ }^{11-14}$ especially if head injury is superimposed. Therefore, a patient on anticoagulant treatment, who has head trauma with any neurological symptoms should undergo CT even in the absence of any neurological deficit.

In the differential diagnosis of the aetiology of vertigo and nystagmus a subdural haematoma compressing the brain stem should be considered. This is especially applicable in the presence of predisposing factors for subdural haematoma. Although this aetiology is uncommon, its simple diagnosis by CT and specific and effective treatment by surgical drainage should be recognised.

1 Cooper PR. Traumatic intracranial hematomas. In: Wilkins RH, Rengachary SS, eds. Neurosurgery. New York: McGraw-Hill, 1980:1659-61.

2 Gudemann SK, Kishore PRS, Miller JD, Girvendulis AK, Lipper MH, Becker DP. The genesis and significance of delayed traumatic intracerebral hematoma. Neurosurgery 1979;5:309-13

3 Schapiro RT. Clinical neurology for the otolaryngologist. In: Paparella $M M$, Shumrick DA, Gluckman JL, Meyerhoff WL, eds. Otolaryngology. Philadelphia: Saunders WB, 1991:2971-82.

4 Munro D. The diagnosis and treatment of subdural haematoma. N Engl F Med 1934;210:1145-60.

5 Horrax G, Poppen J. The frequency, recognition and treatment of chronic subdural hematomas. $N$ Engl $f$ Med 1937;216:381-5.

6 Robinson RG. Chronic subdural hematoma: surgical management in 133 patients. $f$ Neurosurg 1984;61: 263-8.

7 Hernansanz J, Munoz F, Rodriguez D, Soler C, Principe $G$. Subdural hematoma of the posterior fossa in normalweight newborns. $\mathcal{F}$ Neurosurg 1984;61:972-4.

8 Reigh EE, Nelson M. Posterior-fossa subdural hematoma with secondary hydrocephalus. Report of two cases and review of the literature. $\mathcal{F}$ Neurosurg 1962;19:346-8.

9 Kanno M, Shjmosegawa Y, Onuma T. Posterior fossa subdural hematoma in the newborn caused by birth injury. No Shinkei Geka 1989;17:1159-64.

10 Stone JR. Posterior fossa subdural hematomas in newborns (letter). $\mathcal{F}$ Neurosurg 1985;62:626.

11 Gennarell TA. Trauma. In: Yomans JR, eds. Neurological surgery. Philadelphia: Saunders WB, 1990:1978-80 and 2160-8.

12 Fogelhom R, Waltimo O. Epidemiology of chronic subdural hematoma. Acta Neurochir (Wien) 1975;32:

13 Luxon LM, Harrison MJG. Chronic subdural hematoma. $Q \mathcal{F}$ Med 1979;189:43-53.

14 Markwalder TM. Chronic subdural hematoma: a review. f Neurosurg 1981;54:637-45. 